\title{
ABHANDLUNGEN・ARTICLES・ARTICLES
}

\section{L'Etat de droit et la démocratie en Afrique \\ - Le « jurisfascismepositiviste » en question}

\author{
Adalbert Sango Mukalay*
}

\section{Introduction}

En rédigeant ces lignes, je ne m'inscris pas dans la double logique: réactionnaire et révolutionnaire. C'est-à-dire, dans le premier cas, de défenseur de certaines idées reçues par la conservation des idées dominantes sur la conception de l'« Etat de droit » et de la « démocratie »; et « dans le deuxième cas, la remise en question de ces idées reçues en vue d'une ouverture à des nouveautés sur le contenu de ces concepts.

En effet, la question récurrente en matière d'Etat de droit, de droits de l'homme, de démocratie et de droit tout court reste celle de l'existence ou non du droit chez tous les peuples, et ce historiquement parlant. C'est toute la question du sens à donner à tous ces concepts. Car l'histoire du droit nous apprend que le droit a existé sans juriste et sans législateur. ${ }^{1}$ Les juristes compris comme spécialistes de la science juridique ayant été formés dans une académique ou dans une faculté de droit.

Poser la question du rapport entre l'Etat de droit et la démocratie en Afrique revient à s'interroger sur certaines idées reçues relevant d'un débat séculaire sur: Qu'est-ce que l'Etat? Qu'est-ce que le droit? Qu'est-ce qu'un Etat de droit? L'existence, formelle, d'un Etat de droit implique-t-elle nécessairement l'existence d'une culture démocratique? Qu'est-ce que la démocratie? Est-elle une valeur? Une conscience de quelque chose de réel? Est-ce une culture? Quel type de culture?

Autant de questions qui préoccupent tout chercheur en quête d'identité en rapport avec l'expérience du droit ou non dans son histoire. Car, et comme le dit justement Jamil Haddad dans son article sur les mésaventures de la démocratie, "la démocratie a toujours évoqué une idée normative, un idéal plus qu'un système parmi tant d'autres. Un régime démocratique est alors un bon régime émanant de la volonté du peuple et travaillant pour les intérêts du peuple. Cependant autant l'idée est normative et générale autant elle a pu servir à des interprétations différentes; ainsi les 'dictatures du prolétariat, les régimes communistes de transition, se sont définis comme de démocraties populaires, tout comme les ré-

* Professeur Sango Mukalay, Adalbert, Faculté de Droit, Université de Lubumbashi en République Démocratique du Congo.

1 Sacco, $R$. (dir. Par), Il diritto africano, UTET, Turin 1995, pp. 5 et 9. L'éducation juridique dont on parle souvent est une composante de la culture d'un peuple et se reflète dans sa langue (ibidem, p. $11)$. 
gimes occidentaux s'identifient aux principes traditionnels de la démocratie libérale $»{ }^{2}$ D'où l'incomplétude et l'indétermination de l'idée qui sous-tend le concept même de démocratie.

Et quand le professeur Pierre Akele Adau pose la question du défis de la gouvernance et de la démocratie en République Démcra tique du Congo (RDC), ${ }^{3}$ il repose un problème historique, c'est-à-dire celui de l'histoire de la naissance d'un concept, celui de la démocratie et de ses attributs.

Il est connu, au-delà de l'usage idéologique que l'on pourrait en faire, qu'à l'origine, dans le monde grec ancien, la démocratie contenait le principe d'une gouvernance de la cité par les lois faites par les hommes, qui gouvernent les hommes, et non relevant de l'arbitraire des hommes. ${ }^{4}$

L'idéal de la démocratie à l'occidental porte le germe de la libération du peuple du pouvoir d'un seul homme et de "l'autorité économique et morale des classes privilégiées. (...) Et le meilleur garant d'un tel idéal était que le peuple choisisse son gouvernement et ses lois. Mais dès qu'il s'agit de déterminer pratiquement et positivement ces principes, diverses questions se posent: jusqu'à quel point en réalité, dans leur application, ces droits peuvent-ils être les mêmes pour tous les individus? Jusqu'à quel point les principes fondamentaux de la démocratie inséparables en théorie, ne sont-ils pas contradictoires dans la pratique par suite de leur indétermination? Autrement dit, jusqu'à quel point la liberté ne va-t-elle pas à l'encontre de l'égalité? »" Et la liberté des uns ne peut-elle pas être un frein pour celle des autres?

Ce peuple, au nom duquel on parle, comment se définit-il? Est-ce une masse composite de volontés particulières?

Parcourons ainsi certains concepts ou expressions d'importation qui créent une tension en Afrique: ouverture et hostilité ou molle acceptation de la part des Africains. Il s'agit notamment des concepts de droits de l'homme, d'Etat de droit et de démocratie, pour ce qui concerne cette étude.

\section{Les droits de l'homme et leurs ambiguïtés}

L'interrogation sur les droits de l'homme répond à la requête d'un critère universel, indépendant de toutes les sociétés et qui les transcendent, essentiellement déterminé en rapport avec la raison. Celle-ci n'est plus liée à un monde préexistant à l'homme et s'imposant à lui, mais se trouve en l'homme lui-même, ainsi pensé comme naturellement titulaire des prérogatives communes à tous, selon une identification entre l'individu et l'universel.

2 Haddad, Jamil, Les mésaventures de la démocratie, fr.scrib.com/doc/131977757/Philosophie-DuDroit/23mars2013 (consulté le 30 septembre 2014).

3 Akele Adau, P., Défis de la gouvernance, Congo-Afrique, $\mathrm{n}^{\circ} 439$, nov. 2009, 700-714.

4 Bin, R., Lo stato di diritto, Il Mulino, Bologne 2004, pp. 7ss.

5 Akele Adau, P., ibidem. 
Une déclaration de droits de l'homme a une portée universelle, exposant ce que sont les droits naturels de tout homme. Quant à l'acte de déclarer, il a trois significations (Préambule de la Déclaration de 1789): faire connaître des droits ignorés, remémorer des droits oubliés et proclamer des droits méprisés. D'où l'affirmation, en droit: personne ne doit être exclu de ses droits.

Je vais prendre l'exemple de deux droits: égalité et liberté, et ce, en tenant compte du fait que la Déclaration de 1789 est particulièrement et historiquement située; elle porte ainsi en elle une tension entre la contingence historique de son élaboration et l'exigence proclamée d'universalité. Cela suppose que l'universalité, en devenant concrète, puisse se pluraliser. La Déclaration de 1789 porte comme titre « Déclaration des droits de l'homme et du citoyen ». Y-a-t-il une différence entre l'homme et le citoyen? La copule « et »souligne un passage: l'homme doit devenir citoyen, l'homme véritable sera le citoyen effectif.

Mais la question se pose sur le mode de ce passage:

- Pour John Locke, la citoyenneté est le prolongement et le développement d'une humanité initiale. Pour lui, la vie civile affermit, garantit et accomplit la vie de l'homme naturel. D'où déclarer les droits naturels c'est les délimiter et surtout leur donner les moyens de l'effectivité.

- Quant à Jean-Jacques Rousseau, le passage à l'état civil est une réorganisation, ou mieux l'organisation, de la vie humaine. La citoyenneté n'est pas la continuation de la vie humaine, mais elle produit un changement très remarquable (Du contrat social, I,8).

Une instance nouvelle apparaît: la loi positive, qui est l'expression de la volonté générale. C'est elle qui, en société, définit et sanctionne les droits du citoyen. C'est la loi qui, par une transmutation des unités individuelles et absolues en une unité collective et relationnelle, fonde la transition de l'homme naturel à l'homme civil.

Pour revenir à la Déclaration de 1789, pour une pensée de droits subjectifs, la difficulté consiste à résoudre la tension entre la liberté et l'égalité. La liberté, telle qu'elle est pensée par l'esprit moderne, est celle de l'individu, une liberté subjective, comprise comme absolue, comme affirmation abrupte, à la limite une liberté monadique et insulaire. Cette liberté d'affirmation des prérogatives de chacun rend difficile la constitution d'une société. Car comment un élément exempt de relations pourrait-il fonder le lien social? Comment peuton penser le passage de l'individu au citoyen et à l'homme social?

La liberté, ainsi conçue et comprise comme indépendance et désir absolu d'être soi, ne peut fonder la socialité, comme le remarque Thomas Hobbes. Cette vie de pure spontanéité est la vie de l'état de nature. La question est de penser la sortie de soi de l'homme naturel et l'entrée dans un état civil. La fonction essentielle de l'égalité est précisément d'être le principe de ce lien, un lien de droit. On touche par là à l'ambiguïté du concept de droit.

La liberté et l'égalité signifient toutes deux des droits et sont même des droits fondateurs, mais pas au même sens. La liberté est un droit naturel, qualifiant la nature de l'homme indépendamment de tout lien social. Elle est une prérogative absolue et irrécusable, mais aussi une spontanéité incapable de vivre. L'égalité est un droit tout aussi naturel, mais de portée bien différente; en un sens, l'égalité est un corrélat de la liberté dont elle est 
une expression et un prolongement. En effet, si chacun est désir libre et absolu d'être soi, il y a identité, et ainsi égalité, entre tous. Mais c'est justement cette égalité de condition qui devient une opposition à la liberté, du simple fait que la liberté se trouve maintenant posée en relation; et une liberté égale pour tous n'a plus du tout le statut de la liberté absolue et indéterminée. L'égalité remplit ainsi la fonction d'un élément de détermination et de transformation de la liberté: par cette détermination se forme le lien social.

A ce point de l'analyse, l'égalité ne peut signifier qu'une égalité de droit. Les évidentes inégalités de fait s'éclipsent derrière l'égalité de droit, qui est précisément la revendication d'établir l'égalité de fait. L'égalité de droit est essentiellement l'exigence de l'égalisation: la vie sociale doit tendre à l'égalité.

L'important, pour la vie sociale, c'est que l'égalité, antagoniste de la liberté en tant qu'elle met en question son caractère absolu, soit aussi l'élément qui puisse conforter la liberté en l'instituant réellement. Il y a une circularité de la liberté et de l'égalité, telle que l'affirmation d'une liberté abrupte et anomique fait découvrir l'égalité ou au moins l'absence de hiérarchie, et que d'autre part l'égalité établit la liberté comme liberté de relation. C'est en effet de la convention égalitaire que les individus sont censés se passer réciproquement qui institue la liberté comme liberté civile et morale.

\section{Les droits de l'homme: Quels droits? Quel homme?}

Rappelons les caractères majeurs de deux droits sus-évoqués:

- l'égalité, l'individu s'affirme comme être pour autrui. C'est par là aussi que le droit retrouve quelque chose du sens classique qui conduit à la définition de la justice comme égalité.

- avec la liberté est le droit le plus primordial et signifie le pur rapport à soi, l'affirmation de l'unicité de l'individu; elle est l'expression d'un pur être pour soi et d'une simple spontanéité.

Cependant, sans la fraternité (appelé aussi tiers droit) ${ }^{6}$ qui conduit à l'affirmation d'une solidarité de tous les humains, liberté et égalité demeurent face à face, en concurrence. Autrement dit, seule la fraternité peut, dans une doctrine complète des droits de l'homme, fonder le lien social en faisant passer les hommes d'un rapport interindividuel à une société transcendante aux individus. Le droit, comme système positif et objectif d'institutions, requiert la reconnaissance d'une unité entre tous les membres du corps social. La solidarité sociale devient une version politique ou nationale de la fraternité universelle qui constitue le critère ultime.

Sur le plan national, sous une forme ou sous une autre, les textes constitutionnels ou législatifs les proclament dans des formulations variées mais qui, toutes, impliquent que l'homme a des droits opposables à la société et notamment à la société politique et qui, donc, transcendent de quelque façon celle-ci.

6 Clair, A., Droit, communauté et humanité, Cerf, Paris 2004, pp. $66 \mathrm{~s}$. 
Sur le plan international, depuis la Déclaration universelle des droits de l'homme de 1948, les conventions, les résolutions, les organisations se sont multipliées sur la thèse des droits de l'homme.

Les déclarations de principe, fussent-elles dans les formes les plus solennelles, n'impliquent pas qu'elles aient des effets réels sans lacune ou même qu'elles aient, tout court, des effets. Il en va des droits de l'homme comme de la démocratie: tout le monde s'en réclame mais tout le monde ne les pratique pas. Mais l'on sait que, puisque l'hypocrisie est l'hommage que le vice rend à la vertu, elle a du moins le mérite de montrer où est la vertu.

La proclamation des droits de l'homme atteste leur présence dans le développement des institutions positives prenant la forme qui certifie le passage du politique ou du philosophique au juridique.

Les droits de l'homme dont il est question sont la base de la société et non son produit. Ils sont une exigence et non une promesse sans condition. Contenus dans le groupe que constituent les deux précités:

- sont ceux d'être semblables et égaux en dignité;

- ont un contenu variable;

- sont constitués en génération;

- sont justiciables d'émergences successives.

L'idée des droits de l'homme est un héritage intangible, mais non immuable. Ils constituent un fond perpétuel mais ouvert aux dons et legs. Cependant il est à noter que sur le statut religieux, la condition de la femme, la liberté d'opinion et d'information, etc., la position des Etats varie. D'où l'idée des droits de l'homme est liée au droit à la différence de leur conception. De quel homme s'agit-il?

Historiquement, malgré les imperfections, tous les syllogismes et toutes les défaillances, la constitution des droits de l'homme est d'origine religieuse. C'est dans une filiation et ressemblance à un absolu qu'ils prennent racine. Filiation et ressemblance qui renvoient à une création, et donc à un être extérieur à l'homme. Et le premier des droits de l'homme est le respect de la personne humaine.

\section{L'idéologie des droits de l'homme: cas de la France et des Etats-Unis d'Amérique}

Les droits de l'homme peuvent être définis comme le fruit d'un compromis, parfois laborieux, autour des notions de liberté, de dignité, d'aspiration légitime au progrès social. Les droits ne doivent donc pas être vus comme une donnée immuable, imposée aux hommes. Ils sont le fruit d'une histoire complexe, faite d'affrontements et de compromis, entre deux grandes traditions.

Le terroir de l'idéologie des droits de l'homme est interne aux Etats. Elle tend à définir les types de rapports qui existent entre les individus et l'Etat. D'où les droits de l'homme modèlent la société interne, tant par leurs fondements philosophiques que par leur régime juridique établi par les différents systèmes positifs. De leur source interne, les droits de l'homme ont connu un processus d'internationalisation, avec la Déclaration universelle des 
droits de l'homme (1948), la Convention européenne des droits de l'homme (1950) et aussi avec les Pactes des Nations Unies (1966), qui en constituent les jalons.

Il existe en effet une différence entre internationalisation et universalisation des droits de l'homme.

L'internationalisation des droits de l'homme constitue la consécration d'un régime commun particulièrement développé et protecteur. Alors que l'universalisation s'appuie sur diverses conventions à vocation universelle, mais aussi sur l'action militante de nombreuses organisations non gouvernementales, la plupart d'origine occidentale. En prenant l'exemple de deux systèmes juridiques qui ont joué un rôle important dans la consécration des droits de l'homme, le système français et le système américain, la réalité apparaît beaucoup plus complexe et beaucoup plus équivoque.

Ces deux systèmes dominent actuellement le débat idéologique sur les droits de l'homme. La France et les Etats-Unis d'Amérique se présentent comme le berceau des droits de l'homme. Est-ce pour autant qu'ils constituent un socle unique hérité du passé, d'un modèle « occidental » qui aurait vocation à s'universaliser?

Examinons d'abord la conception des droits de l'homme dans ces deux cultures:

Au-delà de la différence du fondement, les contacts entre les Pères Fondateurs et les rédacteurs de la Déclaration de 1789 ont incontestablement existé. Vers la fin du XVIIème siècle, la France et les Etats-Unis d'Amérique ont eu le désir d'écrire une Constitution et d'affirmer les droits de l'homme.

Dans les deux cas, ces derniers sont considérés comme un élément du régime politique mise en place. C'est ainsi que les constitutional rights sont un élément essentiel du constitutionnalisme américain. La démocratie américaine repose sur un double fondement: le régime représentatif d'une part et la garantie des droits d'autre part. Le self government et le respect des droits individuels sont donc les fondements de la légitimité du gouvernement. En France, l'article 16 de la Déclaration de 1789 précise de la même manière que: "toute société dans laquelle la garantie des droits n'est pas assurée, ni la séparation des pouvoirs déterminée, n'a point de constitution ». A contrario, le respect des droits de l'homme et de la séparation des pouvoirs sont les deux éléments qui révèlent un régime qui n'est pas despotique, c'est-à-dire un régime constitutionnel.

Il faut le souligner, là s'arrête le rapprochement entre les deux régimes. La démarche devient différente, dans les deux systèmes, lorsqu'il s'agit des dialectiques entre pragmatisme et dogmatisme d'une part, liberté et égalité, d'autre part.

\section{Le dogmatisme et le pragmatisme}

L'idéologie des droits de l'homme aux Etats-Unis d'Amérique est marquée par le pragmatisme de ses origines: les Pères Fondateurs avaient pour objet essentiel l'affirmation de l'indépendance américaine. La Déclaration d'Indépendance se présente ainsi comme un texte a portée internationale par l'affirmation de l'existence d'une nation aux yeux de l'Angleterre et du monde. 
En France, au contraire, la Déclaration des droits de l'homme et du citoyen apparaît comme un texte plus dogmatique. Il s'agit plus particulièrement de régénérer la société française, et plus largement d'offrir au monde les principes fondateurs de l'édification d'un corps politique légitime. Lorsque les Américains libèrent une nation, les Français édifient une société nouvelle.

\section{- La liberté et l'égalité devant la loi}

Pour les Américains, les droits sont aussi d'essence pragmatique. La finalité poursuivie est de protéger l'individu contre les pouvoirs publics conformément à la tradition anglaise qui, à la proclamation des principes solennels, préfère la mise en place des garanties procédurales permettant au citoyen de se défendre lorsque ses libertés sont mises en cause: la liberté domine, la liberté autonome, qui accorde à l'individu le droit d'être protégé contre toute intervention de l'Etat qui ne serait pas justifiée par la nécessité de maintenir l'ordre ou d'assurer le bien public. L'Etat est considéré avant tout comme un Etat modeste, un Etat peu présent que possible et qui laisse se développer librement l'initiative individuelle.

En France, la liberté ne peut se développer que si elle est accompagnée de la mise en œuvre du principe d'égalité: la liberté doit être acquise grâce à l'égalité de tous devant la loi.

\section{-L'Etat et la liberté d'expression}

En France, la liberté d'expression est considérée comme « un des droits les plus précieux de l'homme » (article 11 de la Déclaration de 1789). Cependant, conformément aux principes du régime répressif, le citoyen peut avoir à répondre de "l'abus de cette liberté dans les cas déterminés par la loi ». D'où l'affirmation du principe selon lequel «la liberté consiste à pouvoir faire tout ce qui ne nuit pas à autrui $»$.

Aux Etats-Unis d'Amérique, la recherche de cet équilibre ne constitue pas une préoccupation. Car la liberté implique avant tout l'abstention de l'Etat qui n'a pas à définir de valeurs. Ce système juridique protège tant la diversité des opinions que celle de ses expressions. Il n'existe pas, aux Etats-Unis d'Amérique, d'ordre public, ou plus exactement d'ordre moral public qui permette d'interdire la circulation de certaines idées. Cela ne signifie pas cependant qu'il soit entièrement impossible de poser des limites à la liberté d'expression.

La contradiction entre les conceptions américaine et française se résument en ceci: pour les Américains la liberté d'expression ne saurait souffrir d'aucune limite; alors que pour les Français, cette liberté est intrinsèquement limitée par elle-même, puisqu'elle ne saurait bénéficier à ceux qui nient les droits de l'homme, et surtout dans leur dimension égalitaire.

L'opposition déjà soulignée entre une conception qui met l'accent sur la liberté et une autre sur l'égalité se retrouve dans Voltaire lorsqu'il dit: «pas de liberté pour les ennemis de la liberté »et « je désapprouve ce que vous dites mais je défendrais jusqu'à la mort votre droit de le dire » (Voltaire). 


\section{- La diversité des moyens d'expression}

En droit français, le 'principe de la liberté d'expression protège l'expression verbale ou écrite d'une opinion, quel qu'en le support. Le droit américain va au-delà de cette analyse et étend la protection à l'expression non verbale.

En effet, brûler, pour un Américain sa carte d'électeur, le drapeau américain ou mener tout autre action plus ou moins provocatrice, n'est en rien sanctionné aux Etats-Unis; alors que ces actions sont susceptibles d'être poursuivies, en droit français, sur le fondement de l'article 431-3 du Code pénal pour lequel tout «attroupement ou rassemblement sur la voie publique ou dans un lieu public susceptible de troubler l'ordre public » peut constituer un délit si les participants refusent de se disperser après les sommations d'usage. Le premier amendement de la Constitution américaine les protège pourtant!

\section{Les droits de l'homme en Afrique ${ }^{7}$}

Devant, apparemment, par beaucoup d'infractions graves et massives perpétrées en Afrique contre les droits humains, beaucoup d'auteurs ont prétendu que «la pratique et même le concept de ces droits sont étrangers à l'approche africaine et islamique de la dignité humaine: elle serait une création de la civilisation occidentale moderne $\gg .{ }^{8}$ Le problème qui se pose est sûrement celui que l'on a de l'acception des droits humains. Et tout dépend du lieu à partir duquel on en parle. Il est toutefois important de s'entendre sur quelques idées ou vérités communément partagées.

Il est séant d'éviter tout romantisme passéiste pétri d'autosatisfaction aveugle. Comme tous les autres coins du monde, l'Afrique n'a pas eu et n'a pas la palme du respect des droits humains. Mais comme les autres continents, avec, bien sûr, des modalités différentes, elle a élaboré, à travers des épreuves et des tourments internes ou externes, des normes et pratiques qui présentent un profil singulier sinon spécifique.

Il n'est pas question, ici, de prétendre donner une liste exhaustive des droits de l'homme en Afrique. Cependant je vais me contenter de les circonscrire autour de trois axes majeurs. Il va s'agir des droits humains à caractère biologique et socio-économique; des droits sociopolitiques et surtout de leurs fondements culturels et éthiques.

Pour la première catégorie, je retiens le respect de la vie et du vivant en général: ils'agit là d'un trait culturel ou sociologique, historique et non racial; d'une exigence de survie collective dans des conditions écologiques, technologiques et structurelles données. Cela est réellement vrai qu'avant de dépouiller un arbre d'une branche ou d'une écorce ou des racines pour en faire un remède, le guérisseur africain se recueillait et lui demandait pardon d'avoir à le mutiler. Le cannibalisme rituel sur lequel l'on a beaucoup disserté, pour néces-

7 Les éléments d'analyse qui constituent toute cette partie, nous les avons tirés d'un savant article du Professeur Joseph Ki-Zerbo publié dans Congo-Afrique, n 430 , décembre 2008.

8 Donnely, J.,Human Rights and Human Dignity. An Analytic Critique of Non-Western Conceptions of Human Rights. The American Political Science Review, June 1982, vol. 76, p. 303. 
sité de survie peut-être, a existé ici ou là en Afrique comme dans tous les autres continents. Mais l'interdiction de verser le sang humain était considérée comme une présomption éminente sanctionnée solennellement, de même en ce qui concerne la torture.

Le droit à la vie implique surtout l'accès à l'alimentation. Dans beaucoup de cas, cet accès est présenté comme un droit direct, immédiat et imprescriptible: par exemple la liberté d'accès gratuit à l'eau et aux puits. Il y a d'ailleurs un rituel éloquent de l'offrande de l'eau à l'étranger et au visiteur. Cela pose la question du droit de propriété caractérisé, le plus souvent en Afrique par plusieurs éléments dont j'évoquerai, surtout et en passant, la dimension communautaire et le caractère d'usufruit qui lui est attaché: "Comment vendre la terre, disaient les anciens, puisqu'elle appartenait à nos ancêtres desquels nous l'avons reçue et que nous la gérons pour la transmettre à nos petits enfants qui en sont les vrais propriétaires? „Ainsi donc, cette norme juridique impliquait un parti pris éthique et même idéologique et religieux, avec des retombées économiques et écologiques évidentes (...).

Par ailleurs et il faut le souligner que le droit à l'hospitalité ne signifie pas le droit à la paresse ni à l'oisiveté. Pour rappel et comme l'indiquait, à juste titre, Julius Nyerere, on donnait à manger et à boire à l'étranger. Mais, les jours suivants, on lui donnait un outil. C'est le fameux système du don et du contre-don, du donner et du recevoir, du droit et du devoir, que les anthropologues ont disséqué à loisir et qui est l'une des structures axiales du comportement sociétal humain partout et toujours.

La place du faible est évidente dans la société africaine. D'où les normes africaines ont souvent un faible pour les faibles. Cela se vérifie déjà dans les contes et les mythes où le plus faible au départ arrive souvent gagnant. Il y a bien sûr les personnes âgées qui ont une place prépondérante au point de verser parfois dans la gérontocratie. C'est que le droit des vieillards se fonde dans le fait qu'ils sont non pas des déchets d'humanité, mais qu'ils ont accompli l'humanité. C'est pourquoi on dit, en Bambara: « Le vieillard vaut mieux que son prix ». Car, en l'occurrence, il s'agit non de la valeur d'échange, mais d'une autre échelle de valeur. " "Je juge une civilisation par la manière dont elle traite les vieillards », dit encore Julius Nyerere. Car «c'est la vieillesse qui est puissance ».

Quant aux droits des enfants, l'enfant était désiré, au point de faire de la stérilité une malédiction. "C'est par les enfants que nous sommes immortels », disait-on. Là aussi, la Traite des Noirs a constitué une étape catastrophique pour les droits de l'enfant africain.

Pour les femmes, il faut le reconnaître, leur sort est loin d'être idéal. En Afrique comme ailleurs, la face féminine de l'effigie humaine est vraiment le revers de la médaille. Non pas seulement par les aspects les plus «médiatisés » comme l'excision de certains organes génitaux, le labeur écrasant et sans fin, en particulier par portages innombrables, mais aussi par la place subordonnée qui semblait lui être affectée dans la société. ${ }^{10}$ En matière de virginité

9 Plus quelqu'un est âgé, plus grande est la part qu'on lui réserve »: proverbe Mongo (République Démocratique du Congo).

10 De manière systématique dans la société africaine ancienne, car aujourd'hui quelques évolutions notables sont palpables dans beaucoup de villes du continent noir. 
avant le mariage et d'adultère ${ }^{11}$ par exemple, les droits et les devoirs des femmes sont extrêmement variés selon les ethnies et les groupes sociaux.

Mais il serait aisé de faire de la condition de la femme africaine une lecture alternative qui montrerait que par certains côtés, dans de nombreuses sociétés sub-sahariennes, la femme avait un statut bien moins déplorable que dans d'autres continents.

A l'égard des malades, et en particulier des fous, l'option africaine, contrairement à celle qui prévaut dans maints pays du Nord, c'est le refus de l'exclusion, le droit à la présence, le refus de laisser le malade seul. C'est pourquoi le modèle d'hôpital-isoloir-mouroir a été rejeté et transformé en Afrique par l'invasion des bien-portants. Beaucoup d'explorateurs européens tombés malades ont bénéficié de soins acharnés jusqu'à la guérison de la part des femmes et des guérisseurs africains.

C'est pourquoi les droits des malades sont mieux défendus sur ce continent que dans beaucoup de pays très riches. La santé n'a pas de prix. La maladie corporelle n'est qu'un signe d'un déséquilibre plus profond mais invisible qui perturbe la sphère de l'esprit, de la société, voire de l'univers cosmique. C'est à l'interface de la trilogie: malade, guérisseur, milieu naturel que la santé doit être recherchée. L'option africaine de la thérapie a consisté à réunir tous ces éléments dans une totalité où la nature est tour à tour conjurée et associée, où l'ensemble de la communauté est investi de la mission curative, même si certains en sont les spécialistes.

Quant aux fous ou ceux qu'on baptise tels, il suffit de citer un dicton commun à plusieurs langues africaines pour poser le principe de leur non exclusion dans des espaces spécialisés: «Le fou est une personne (humaine) folle! »D'où la pratique correspondante; le fou, en cas de folie douce, est membre à part entière de la société qu'il va distraire ici et là par des remarques peu orthodoxes. S'il s'agit d'un fou furieux, on lui leste la jambe avec une bille de bois qui, sans l'immobiliser, ralentit sa marche et l'empêche de nuire à autrui. Ici encore, on voit l'équilibre qui préside à l'approche africaine des droits humains.

La liberté d'expression est loin d'avoir été toujours assurée en Afrique. C'est ainsi que sous le roi du Sosso (à la jonction de la Guinée, du Sénégal et du Mali), redoutable prince réfractaire à l'islam, au début du XIIème siècle, la terreur qu'il inspirait était telle que, lorsqu'on voulait le critiquer, l'on cherchait une grande gourde et l'on y introduisait la bouche pour y exprimer et y enfermer à la fois son opinion. C'est en libérant le Manding de ce joug abrutissant que Sundjata aurait acquis le renom qui, jusqu'à ce jour, auréole son souvenir.

En effet, l'organisation de la parole publique, bien que privilégiant certaines catégories (anciens, hommes, clans princiers, etc.), ménageait à la plupart des groupes un espace de contribution. C'est l'associationnisme africain qui préférait impliquer tous les groupes s'exprimant comme tels plutôt que de risquer l'éruption de forces centrifuges qui eussent été alors difficiles à contenir. Ce schéma était vérifié surtout dans les sociétés villageoises où

11 Ici aussi, il faut le reconnaître, c'est surtout l'adultère de la femme qui est plus mis en exergue et qui peut avoir des conséquences, même juridiques, comme la séparation ou le divorce, par exemple. 
l'accès à la parole publique était plus immédiat et plus ouvert. A ce niveau, pour chaque famille ou clan, la parole était non seulement un droit mais un devoir auquel on ne pouvait se dérober. Mais même dans les hégémonies impériales africaines de la grande époque, certaines structures sociales permettaient aux catégories dominées de proférer, en certaines circonstances, des critiques ou des censures qui exprimaient la pensée et le sens de l'opinion publique. Les clans ou « castes » les plus éloignés de la noblesse avaient le plus grand accès à la parole.

La liberté d'association était largement assurée et se manifestait dans une infinité de groupes et confréries de travail, de loisirs, d'activités artistiques, initiatiques ou ésotériques, de « classes d'âge » et dont certains, comme le « ton » villageois, ont été revitalisés de nos jours pour dynamiser l'essor socio-économique.

La liberté de déplacement était et demeure l'un des droits les plus populaires du continent africain, se soldant parfois par des fraudes commerciales massives à travers les passoires qui constituent les frontières artificielles entre les pays. Ce n'est pas aujourd'hui seulement que l'Afrique est le continent des réfugiés. Déjà au niveau familial, les femmes africaines, surtout du fait du maintien de leurs liens (y compris le nom) avec leur famille d'origine, ne se font pas faute d'exploiter le droit à rejoindre cette famille en toute circonstance: à temps, voire à contretemps. Mais le droit au déplacement, c'est aussi et surtout la liberté de « voter avec ses pieds » pour échapper aux aléas de la vie: tyrannie du pouvoir, épidémies, surpeuplement, etc.

La carte ethnique, en forme de puzzle inextricable, est la matérialisation concrète de ce mouvement de groupes sociaux de toute taille et de toute extraction. Le droit d'asile et le droit à la terre au lieu de destination constituaient le facteur attractif complémentaire des facteurs répulsifs cités plus haut.

L'immunité protégeait aussi et surtout les envoyés, ambassadeurs et négociateurs. Le principe était que le mandataire chargé de délivrer un message, oral ou écrit, n'était pas responsable du contenu du message et ne saurait être sanctionné au cours et/ou du fait de son mandat. D'où le dicton: «La foudre ne tombe pas sur un envoyé, sur un ambassadeur ».

Je chute, enfin, après ces quelques cas, sur la liberté religieuse. Elle est un des traits caractéristiques de l'évolution africaine. Il y a eu, bien sûr, des conflits liés aux esprits, génies ou « dieux » divers et concurrents de la religion traditionnelle. Mais celle-ci, n'ayant pas de dogme révélé à préserver comme dans « les religions du livre », laissait beaucoup plus d'initiatives à ses adeptes pour les rituels liturgiques, les initiations et innovations; tout en gardant un redoutable potentiel de résistance qui n'est pas encore épuisé jusqu'à nos jours.

Pour l'existence des droits de l'homme, les fondements culturels et éthiques sont incontournables. Car Il n'y a pas de droits humains sans fondement éthique, le droit lui-même se situant à l'interface de la force et de l'éthique, comme une résultante de ces deux éléments souvent conflictuels. Entre l'exigence de l'idéal humain et la violence des intérêts, il y a le droit. Cette éthique africaine se fonde avant tout sur l'éminente dignité de la personne humaine, laquelle est fondée dans les mythes les plus anciens. 
La deuxième valeur axiale qui fonde les droits humains, c'est la solidarité, vertu africaine si profondément ancrée dans les pratiques et les consciences qu'elle finit par inhiber les efforts de développement par certains effets induits. Mais il est clair que, comme fondement des droits humains, la solidarité constitue une base beaucoup plus solide que l'individualisme égoïste.

Une autre valeur à laquelle les sociétés africaines sont fort sensibles, c'est la justice que les gens privilégient presque même à la liberté, avant de s'apercevoir que, sans liberté, il n'y a pas de justice.

Derrière ces valeurs qui habitent les tréfonds de toute conscience humaine lucide et motivée, il y en a d'autres sur lesquelles les Africains ont mis un accent singulier. Par exemple, la pitié qui n'est pas du misérabilisme ou de la sensiblerie niaise, mais l'élan de compassion qui mobilise les autres valeurs au service des droits humains. La pitié est une valeur démocratique en dehors de laquelle la jungle n'est pas loin. Enfin, c'est la tolérance, l'acceptation presque inconditionnelle de la différence qui implique qu'on est prêt à défendre farouchement sa propre différence et son identité.

Il existe, en Afrique, des cadres qui garantissent et compromettent, en même temps, la protection des droits de l'homme. En sociologie et en politique, il existe toute une série de mots et de concepts qui encombrent la réflexion et donc l'action en Afrique. Car il s'agit des concepts transférés pour rendre compte de réalités éloignées dans l'espace et dans le temps. D'où un double risque méthodologique d'européocentrisme et d'anachronisme: individu, Etat, nation, ethnie, peuple, tribu, Etat de Dieu, démocratie, etc. Les réalités africaines sont casées de force dans des moules préfabriqués dont il faut retenir seulement certains, dans une lecture critique, en évitant de se dérober sur l'immense sujet des droits des peuples dans l'histoire africaine.

Faut-il le rappeler que l'État bureaucratique et oppresseur n'a émergé, en Afrique au Sud du Sahara, que très tardivement en raison de plusieurs facteurs inhibiteurs, parmi lesquels on peut citer:

- la rareté des véhicules à roue, qui limite aussi la centralisation; l'apport tardif des armes à feu, qui ont puissamment contribué à l'absolutisme étatique;

- la rareté de l'unification linguistique autoritaire;

- la rareté de l'imposition d'une religion à ambition universelle.

Le principe fondamental du système africain, plus ou moins inspiré par les réalités, et nonobstant les nombreuses exceptions, c'était donc la socialisation du pouvoir politique et économique. L'Etat, c'est-à-dire l'appareil qui met en œuvre le pouvoir a été souvent réduit au minimum malgré les apparences gérontocratiques dans les villages et autocratiques dans les royaumes et empires. Par exemple, très rarement l'espace étatique a été identifié à une espèce ethnique. Presque toujours, le pouvoir africain était inter et trans-ethnique par un brassage énorme de peuples.

L'un des postulats qui ressort de l'analyse historique, c'est la recherche par les « constituants » africains de l'équilibre entre les acteurs politiques grâce à la mise en œuvre de trois grandes normes: la limitation du pouvoir; le partage et la réalisation d'un Etat de droit. 
Alors que dans L'Esprit des Lois, Montesquieu écrit: «Il faut que le pouvoir arrête le pouvoir ", les Africains avaient une nette conscience de la charge de violence potentielle incluse dans le pouvoir puisqu'ils l'ont baptisé « la force ». C'est pourquoi, sur injonction des gens du peuple, dans des serments solennels d'investiture, les futurs chefs s'engageaient à ne pas abuser du pouvoir.

C'est après ce serment que le peuple, à son tour, par ses représentants, prêtait le serment de le servir et de l'aider à bien gouverner. Il s'agit bien là d'une sorte de contrat synallagmatique, d'une constitution. Car ces serments pouvaient, par la suite, être invoqués contre les parjures. Très souvent, la procédure de plainte, de doléances vers le niveau supérieur était prévue, allant du village (de la colline, au Rwanda et au Burundi) jusqu'au roi, en passant par les chefs inférieurs et supérieurs (chefferies, provinces).

La conception africaine s'éloigne de la plupart des systèmes politiques africains d'aujourd'hui où« pouvoir »égale « avoir »et réciproquement.

Situation différente aussi du cas des pays industrialisés où le pouvoir est recherché après que l'avoir été largement assuré. Le chef politique n'a pas pouvoir sur la terre et sa dévolution. La compétence politique et judiciaire n'est pas foncière. Cette dernière revient aux chefs de terre, souvent descendants des premiers occupants. En tant que Chef, il devait rigoureusement redistribuer les biens qu'il recevait et non les accaparer, cela contribuait aussi à limiter son enrichissement.

La meilleure façon, dit-on, de limiter le pouvoir, c'est de le partager. Tel était le second principe selon lequel la meilleure garantie du pouvoir était d'en multiplier les parties prenantes en associant le maximum de groupes.

L'armée de métier, constituée très tardivement, était avant tout, jusqu'au XVIIIème siècle, presque toujours un outil de conquête non point du pouvoir mais de territoires extérieurs. Souvent, le roi est élu par de grands électeurs non nobles, afin d'observer un principe de neutralité entre les clans nobiliaires en compétition.

L'Etat africain, dans de très nombreux cas, méritait l'appellation d'Etat de droit. Le droit ici était dit par la coutume. Le roi, loin d'être au-dessus de la coutume, devait en être le premier serviteur, prisonnier qu'il était d'usages minutieusement codifiés.

Dans maints royaumes africains, le roi était tellement subordonné au peuple que, lorsqu'il s'affaiblissait, on estimait que cela pouvait nuire à la prospérité de son peuple et on l'éliminait rituellement. Ce régicide constitutionnel pouvait intervenir aussi en cas de trahison des normes de la coutume (Yoruba).

Dans les sociétés à pouvoir diffus, la socialisation était encore plus grande à travers les groupes d'âge, la parenté et les cultes religieux.

Quel est l'impact des systèmes extérieurs qui se sont imposés à l'Afrique? En quoi ontils influé sur l'évolution des droits humains sur le continent? Sans vouloir intenter des procès faciles ni dresser des bilans unilatéraux, il faut reconnaître que l'Afrique noire a payé un lourd tribut sur ce plan et que les régimes établis par les étrangers, avec souvent la complicité des partenaires africains, ne compteront pas dans les Annales de l'Histoire universelle comme des modèles de protection des droits de l'être humain. 
De la colonisation à nos jours, l'Afrique noire n'a jamais recouvré son indépendance réelle. La Traite des Noirs fut motivée par l'intégration de la force de travail négro-africaine dans les circuits du mercantilisme capitaliste: ce fut l'un des épisodes les plus sinistres de l'avilissement de l'homme. Le régime sud-africain de l'apartheid aété très absolu en matière de droits de l'homme, un épilogue affreux et constitutionnel de la Traite des Noirs. La colonisation, elle, fut motivée, disait-on, par la nécessité de mettre fin à l'esclavage en Afrique. Mais pour restaurer l'Afrique, elle écrasa de nombreux Africains. C'est le sens du « double mandat » (de civilisation et d'exploitation) énoncé avec le plus d'éclat par le Britannique Lord Lugard.

Ceux de la génération du Professeur Ki-Zerbo, qui a lutté avec une farouche énergie pour l'indépendance, croyaient fermement que celle-ci instaurerait des cadres stables pour la défense et l'illustration des droits de l'homme en Afrique. On connaît la suite qui n'en finit pas d'écœurer le monde et l'Afrique: tyrannies cannibales (chez Bokassa, écrit-on!), dictatures ouvertes ou larvées et masquées (dans nombres de pays africains jusqu'à nos jours), crues et cruelles, ou couvertes des façades démocratiques (pour ceux que l'on qualifie des modèles de bonne gouvernance mais une main lourde sur les libertés individuelles), conflits internes ou guerre de frontières (dans beaucoup de pays africains portes-étandar de l'extrême pauvreté de leurs populations), guerres civiles et génocides, exodes d'apocalypse de peuples entiers évacuant en bloc leur espace abandonné aux seigneurs de la guerre, etc.

Le tableau des droits de l'homme dans l'Afrique précoloniale, auquel on recourt pour dépeindre un peu cette Afrique, peut paraître partiel et partial. En fait, il ne s'agit pas d'autosatisfaction ni de romantisme rétrospectif et subjectif, selon le dicton: « Quand mon ami est borgne, je le regarde de profil! » Le droit le plus impérieux pour les Africains, aujourd'hui, est le droit de se connaître et d'être connus. « Le droit et le devoir... ». Qui sommes-nous réellement? Avons-nous une identité ou sommes-nous- comme l'a dit quelqu'un -un canevas mouvant d'identités partielles acquises au gré de circonstances contingentes? ${ }^{12}$ En effet, le mal fondamental qui mine tous les efforts pour redresser l'Afrique et prévenir les attentats contre les droits de l'homme et donc qui aboutit la non-existence d'un Etat de droit, c'est la désintégration générale et incontrôlée des éléments organiques de notre identité collective.

Le premier droit et devoir, c'est de bâtir de nouvelles cohérences, une synthèse historique pour un nouveau départ en évitant deux écueils graves et dangereux pour les droits de l'homme. D'abord, la fuite en avant vers l'intégration-désintégration, l'intégration suicidaire dans l'Ordre mondial, dans le marché libre (pour les autres!) en effaçant l'État tué dans l'œuf avant même que d'éclore.

Autre dérive grave et majeure: c'est, contrairement à la synthèse nécessaire, le mimétisme du droit public et privé. De même, des groupes ethniques identiques qui obéissent au même droit originel sont souvent assujettis, du fait du partage colonial, à des législations différentes depuis les indépendances. A cet égard aussi, le mythe du droit traditionnel

12 Billier, J.-C., et A. Martyoli, Histoire de la philosophie du droit, Armand Colin, Paris 2001, p. 16. 
comme entrave et frein à la modernisation doit être-dénoncé. Un droit " moderne suffit-il en lui-même à moderniser un peuple? Comme dit le proverbe: On peut amener de force un âne au puits mais on ne peut le forcer à boire ».

L'individu africain consacré sujet du droit à la place du groupe social d'antan n'en devient pas automatiquement un acteur social et politique réel. C'est pourquoi l'Etat africain peut se féliciter de ce que la société civile obéit toujours à quelques normes traditionnelles qui assument certaines charges énormes à caractère de service public (santé, sécurité, éducation, information, etc.). L'Etat utilise à fond les rouages traditionnels et informels de la société civile sans leur reconnaître les droits y afférents et en les laissant dépérir à petit feu.

S'il faut refuser la fuite en avant consistant à se réfugier dans le particulier des autres baptisés Universels, il faut aussi rejeter la fuite vers un passé qui ne vaudrait que comme patrimoine momifié. Un puissant effort de refondation collective et de création des conditions d'un essor économique et social s'impose si l'on ne veut pas continuer à mendier pour survivre. Or, ceux qui mendient n'ont qu'un seul droit: recevoir. Et « la main qui demande est toujours en dessous » (Félix Houphouët Boigny) même si nous sommes exportateurs nets de capitaux.

Partout dans l'histoire, l'Etat et/ou la société civile surtout, de gré ou de force, ont été les artisans de l'essor des droits de l'homme. La société civile africaine (femmes, jeunes, paysans, intellectuels, syndicats, cadres, opérateurs économiques) a déjà bien mérité de ce noble combat.

Mais ce qui reste à faire est encore plus considérable! Cette tâche sera facilitée en dépassant l'Etat par le bas, par décentralisation vers les communautés de base, foyers puissants de défense et de sauvegarde des droits humains concrets et de la démocratie au quotidien. Il importe de dépasser l'Etat également vers le haut, vers des structures fédérales, seules capables d'exorciser le spectre de la dictature.

\section{Le concept de démocratie: bref historique}

Selon le Dictionnaire des Droits de l'homme, ${ }^{13}$ " l'idée de démocratie fait d'un consensus, mais celui-ci repose sur une ambiguïté. Au sens traditionnel de gouvernement par le peuple actualisable selon divers modèles, s'oppose la tentation de réduire la démocratie aux droits de l'homme».

Il est clair que cette affirmation met en exergue les origines culturelles du concept même de démocratie et de ses principes. Et c'est toujours dans le monde occidental où il y a eu des oppositions à cette idée, bien qu'ayant pleinement son acception étymologique de « pouvoir du peuple».

A ce stade, une analyse s'impose! Quand on parle du peuple, de quel peuple s'agit-il? S'agit-il du peuple représenté par une élite qui gouverne en son nom? Et que signifie gou-

13 Dictionnaire des droits de l'homme, p. 262. 
verner? Le Dictionnaire précité ${ }^{14}$ essaie d'y répondre: c'est « (...) le fait de prendre des décisions collectives, non au sens de décisions prises collectivement, mais de décisions qui concernent la collectivité et de décisions qui s'imposent à tous. Il s'agit de décisions d'une minorité conventionnellement acceptées comme volonté du peuple ».

Par conséquent, le prototype de la meilleure gouvernance est cette forme de gouvernement de la majorité par une minorité.

Mais que dire alors de la classification, par les anciens, des régimes politiques?

Platon et Aristote, en considérant l'Etat en relation avec l'accomplissement du bien commun, distinguent les constitutions possibles en constitutions justes et injustes:

JUSTES

- la monarchie: gouvernement d'un seul qui protège le bien de tous

- l'aristocratie: gouvernement des vertueux qui protègent le bien de tous sans s'attribuer des privilèges

- la république: gouvernement populaire qui protège le bien de toute la cité

\section{INJUSTES}

- la tyrannie: gouvernement d'un seul poursuivant son propre intérêt

- l'oligarchie: gouvernement des riches qui cherchent le bien économique personnel

- la démocratie: gouvernement de la masse populaire qui veut supprimer toute différence sociale.

Chez les philosophes de l'époque moderne ces hypothèses connaissent une inversion de tendance par rapport à celles de l'antiquité et du Moyen âge: pendant que les derniers retenaient la monarchie absolue comme forme idéale du gouvernement, les premiers se sont faits portes-étandar de la monarchie parlementaire et de la république.

Aujourd'hui c'est la forme républicaine qui est considérée comme la plus adaptée à la protection des droits et l'accomplissement du bien commun.

D'où, en rapport avec les modèles de démocratie, celle représentative, pour qu'elle « reste démocratique, il ne suffit pas que les représentants agissent au nom des représentés, il faut aussi qu'ils répondent, jusqu'à un certain point, à leurs souhaits $\gg .{ }^{15}$ Car l'on constate, notamment en Afrique en général, que la représentation nationale ${ }^{16}$ se mue en caste au-dessus des lois qu'elle vote. Pour ne prendre qu'un exemple, en République Démocratique du Congo les élus votent des lois qu'ils sont les premiers à ne pas respecter. C'est le cas notamment du respect de l'obligation de payer des impôts et taxes à l'Etat. Cela pourrait être qualifié de comportement non démocratique, au sens de valeur.

\section{La notion d'Etat de droit}

La notion d'Etat de droit est l'une parmi les plus controversées de la science du droit de ces derniers siècles.

14 Dictionnaire des droits de l'homme, p. 264.

15 ID.,op. cit., p. 266.

16 Députés tant nationaux ou régionaux que provinciaux. 
" Concept fondateur du droit public moderne, l'Etat de droit traduit une certaine vision du pouvoir, forgé au fil de l'histoire de l'occident et qui apparaît comme inhérente à la conception libérale de l'organisation politique: donnant à voir un pouvoir limité parce qu'assujetti à des règles, il implique que les gouvernants ne soient pas placés au-dessus des lois, mais exercent une fonction encadrée et régie par le droit. La diffusion actuelle du thème témoigne que cette représentation s'est désormais mondialisée, l'Etat de droit devenant la caution de la légitimité de tout pouvoir $\gg .{ }^{17}$

Comprenant une signification originaire, une inflexion progressive et des implications nouvelles, ${ }^{18}$ c'est sa diffusion internationale qui intéresse mon étude.

Dans son sens large, ce concept peut être compris comme un organisme politique qui a sa légitimité d'une constitution protégeant les droits fondamentaux du citoyen et non de l'arbitraire du pouvoir d'un souverain. Ainsi circonscrit, l'Etat de droit établit une distribution de pouvoir entre ses différents organes. ${ }^{19}$

Le concept renvoie, il faut le rappeler, aussi à la tradition philosophique du « gouvernement par les lois »en opposition à celui des hommes circonscrit dans sa double dimension ou signification d'un gouvernement per leges et d'un gouvernement sub lege.

Beaucoup sont des théories, d'ailleurs différentes, sur l'Etat de droit. D'où de l'expérience de ces théories, je peux tirer ce qui suit:

Un Etat de droit, et cela est commun à tous les pays remplissant ces critères, est caractérisé par:

- Le principe de diffusion du pouvoir, qui établit l'égalité juridique (tous les individus étant considérés égaux devant la loi); l'unicité du sujet de droit; la certitude des droits (tous les citoyens devant connaître leurs droits et les lois en vigueur ${ }^{20}$ ); la reconnaissance constitutionnelle des droits subjectifs (cfr Titre II de la Constitution de la RDC du 18 février 2006).

- Le principe de différenciation du pouvoir, qui établit une délimitation de la sphère d'exercice du pouvoir politique (ce dernier devant être autonome par rapport à celui éthico-religieux et économique); une séparation de pouvoirs; une subordination du pouvoir législatif en rapport avec le respect des droits sanctionnés dans la Constitution; une autonomie du pouvoir judiciaire. Les éléments sus-évoqués clôturent les aspects communs qui fondent les théories sur l'Etat de droit.

Quant aux expériences historiques, il sied de relever quelques exemples:

- L'expérience anglaise de la «Rule of Law »: Dans la conception anglaise de l'Etat de droit, la souveraineté appartient au Parlement (legislative sovereignity composé de la

17 ID., op. cit., p. 388.

18 ID., op. cit., pp. 388-389.

19 Ibid.

20 Cependant dans beaucoup de pays africains, l'on note une carence de l'information législative des citoyens. Et, ce, pour plusieurs raisons. 
Couronne, la Chambre des Lords et celle des Commons) et exerce son primat normatif presqu'exclusivement envers le pouvoir exécutif.

Les principes de base sur lesquels s'édifie ce type d'Etat de droit sont à trouver dans l'égalité juridique des sujets, égalité posée indépendamment du rang du citoyen anglais et de ses conditions économiques; dans la protection des droits subjectifs, principalement assurée par la juridiction des Cours des commonlaw, et enfin dans la synergie normative entre le parlement et les Cours judiciaires.

En effet, bien que les Cours ordinaires n'aient aucun titre pour syndiquer les actes du parlement et ne puissent être considérés comme garantie de la constitution, elles ont le devoir d'appliquer la loi dans le respect des précédents judiciaires et ont la faculté d'interpréter la loi selon des cas.

L'Etat de droit anglais est privé d'une constitution écrite. Cette dernière se réduisant, en effet, à l'ensemble des traditions judiciaires, des actes normatifs et des pratiques sociales qui ont limité le pouvoir exécutif dans l'histoire anglaise.

- L'expérience allemande du «Rechtsstaat »(Robert von Mohl): Le Rechtsstaat allemand concentre les attributs de la souveraineté sur le pouvoir législatif qui jouit ainsi d'un primat normatif absolu sur les autres pouvoirs.

La protection des droits subjectifs est confiée au parlement. Le primat de la loi se traduit par le 'principe de légalité'.

- L'expérience française de l'Etat de droit a son origine dans la Révolution de 1789 avec laquelle était fondée la théorie de la souveraineté et du parlement en tant qu'organe dans lequel pouvait s'exprimer la volonté peuple.

La première théorie, explicite, de l'Etat de droit en France remonte à Raimond Carrè de Malberg, théorie qui prend ses distances de celle des Jacobins français et tend à limiter les pouvoirs du Parlement de manière à éviter que le pouvoir constituant (volonté générale) et le pouvoir constitué ne soient confondus en lui.

En effet, selon la théorie de Raimond Carrè de Malberg, le Parlement doit être subordonné au droit en tant qu'il est un pouvoir constitué, et ses fonctions doivent être soumises aux limites et contrôles, et les citoyens doivent se doter des instruments légaux pour s'opposer à la volonté du législateur quand ce dernier viole leurs droits.

\section{La démocratie comme valeur}

De ce qui précède, il ressort que l'accent, dans la conception d'un Etat de droit, est mis plus sur l'instrument normatif comme garantie de l'autonomie de l'individu face au pouvoir et à la société. Et par ricochet, l'idée même d'« égalité » n'était pas fondée sur celle des l'« égalité des droits »définissant cette autonomie.

Avant de se fonder sur le droit, comme rappelé par les anciens, la démocratie doit être comprise comme une « valeur », et une « valeur positive ». ${ }^{21}$

21 Haddad, J., op. cit., p. 6. 
Le même Jamil Haddad ajoute que «la démocratie ne veut rien dire si elle n'est pas définie à partir de (sic) principes clairs traduisant des valeurs bien déterminées et des rapports réels entre l'individu et la société. (...) La démocratie est un système politique et, à ce titre, elle ne peut être en soi un but, un idéal, c'est un cadre à l'intérieur duquel fonctionne la société ».22

Ces affirmations me renvoient à un questionnement en rapport avec la conception de la démocratie dans des pays en développement ou plus spécifiquement dans des pays postconflits. Et à ce stade des questions, à mon avis, doivent être soulevées.

Il est des situations que l'histoire du droit nous apprend que des systèmes qui considèrent que l'individu se définit par rapport à son appartenance à la société, ce qui le met en situation de subalternité (même juridique) par rapport à cette dernière, « reconnaissent à la société une réalité spécifique et un intérêt qui transcendent les intérêts particuliers. Le système démocratique libéral, qui suppose l'égalité des droits des individus, a de la difficulté à établir que l'intérêt exprimé par le plus grand nombre soit l'intérêt général de la société, surtout si celle-ci est envisagée du point de vu du progrès et en fonction d'un idéal. Non seulement la démocratie a abouti, de fait, à sa propre négation dans les réactions communistes et fascistes, mais encore, faute d'un idéal positif, elle peut aller à l'encontre de ses propres valeurs ou à la limite, aboutir à la dissolution de l'Etat et même à la désintégration de la société civile. Ceci se produit lorsque la société n'est pas prémunie matériellement et moralement contre les forces irrationnelles qui peuvent y déterminer le jeu démocratique et décider du sort de la population. Ou encore lorsqu'elle n'admet pas que tout le monde ne peut pas avoir raison à la fois et que la raison peut aussi bien être la 'raison' de la force ou de la ruse $\gg .23$

En Afrique, en particulier, il existe des valeurs dont le respect constitue l'expression de l'Etat de droit et de l'idée basique de démocratie. C'est notamment la famille, la spiritualité et l'éducation. ${ }^{24}$

En effet Ghislain Kitenge Mpange, parlant de ces valeurs, stigmatise les tares qui y sont continues et qui expliqueraient, aujourd'hui, cette difficulté d'ouverture de l'Africain vers l'autre. Et pour ne prendre que l'exemple de la famille, "l'épouse, quelle que soit son instruction, considérera le foyer conjugal comme une terre étrangère et temporaire, car son foyer véritable reste celui de ses parents. $»^{25}$

22 Ibid.

23 Ibid.

24 Kitenge Mpange, G., Le double agenda de la R-D Congo: Tribus ou Nation?, L'Harmattan, Paris 2007.

25 ID., op. cit., p. 58. En effet, ces anomalies comportement des conséquences sur le plan social et politique d'un pays. 


\section{La démocratie comme conscience}

La démocratie devrait se définir ou mieux être comprise comme un cadre de jeu. Car tant que les cadres agissant, en tant que gouvernants, n'auront pas intériorisé le sens même de leur action, pour laquelle ils se réclament être des représentants du peuple, c'est-à-dire pour la défense des biens relevant de l'intérêt général, ils ne peuvent, en aucun cas, prétendre au bon fonctionnement du système démocratique.

Et l'auteur précité de confirmer: «Si les moyens ne sont pas légitimés et animés par les fins, la démocratie devient un système où prédomine la réclamation des faibles et le règne de la médiocrité ou encore tout simplement la loi du plus fort. $»^{26}$

Pour corroborer mon analyse sur la démocratie comme conscience, je note une certaine mésaventure de la démocratie dans son acception occidentale en Afrique. En effet, « (...) dans les pays non préparés (sic) à la démocratie, par suite de leurs conditions économiques, sociales et culturelles, la démocratie n'est qu'une façade (sic). On y trouve la dépendance de la population, aussi, à l'égard des puissances économiques dominantes mais spécialement à l'égard des pouvoirs traditionnels particuliers, dans certains cas, à l'égard d'un pouvoir religieux qui refuse de dissocier ce qui est à César et ce qui est à Dieu. ${ }^{27}$

De toutes les façons, il faut noter que dans cette situation, des forces spécifiques trouvent dans les règles fondant la démocratie les conditions propices pour un jeu, libre en soi, mais qui n'a rien de démocratique. D'où la situation d'un certain monopole de l'élite en défaveur de l'intérêt général. ${ }^{28}$ Cela implique l'inexistence d'une vérité absolue, conçue comme valeur, et l'émergence d'une idéologie, quand bien même non formellement affirmée et assumée, d'une vérité relative, responsable, de mon point de vue, de beaucoup d'indéterminations, sur le plan théorique, du concept même démocratie.

De manière pratique, pour dépasser cette étape, il existe un impératif, c'est l'assurance dans les conditions de développement d'une liberté psychologique et surtout morale des individus; la dernière conséquence positive consiste dans le développement des qualités morales et culturelles de la population en plus des conditions économiques et juridiques.

La démocratie n'est pas liée au dogme préétabli consistant dans la foi en l'individu. Elle se focalise plus, et indépendamment de la culture qui la développe, sur les libertés liées aux individus tout en développant la raison en l'homme en vue de mériter la dignité d'être libre et justifier ainsi la souveraineté attribuée au peuple.

26 Kitenge Mpange, G., op. cit., p. 56.

27 Haddad, J., op. cit., p. 6.

28 Sango Mukalay, A., L'Etat de droit comme condition du développement en RDC, in: Konrad Adenauer Stiftung (ed.), Démocratie, décentralisation et économie sociale de marché en République Démocratique du Congo, Konrad Adenauer Stiftung, Kinshasa 2013, pp. 19-30. 


\section{La démocratie comme culture}

Souvent quand on parle de démocratie ou d'Etat de droit, on parle des gouvernants ou des gouvernements et non pas de l'état des personnes. Car, en Afrique, l'on s'autoproclame facilement démocrate. Et beaucoup de démocraties sont dans des oppositions politiques aux régimes régnant. Par le jeu d'alternance, si ces dits « démocrates » accèdent au pouvoir, ils deviennent, par la force des choses, des « dictateurs ». Comment expliquer cela? Par le manque d'une culture politique démocratique, qui accepte l'autre dans sa diversité et qui apprécie ses apports positifs à la société. ${ }^{29}$

L'idiotie, si je me peux me permettre ce terme, vient d'une mauvaise information sur la réalité des faits et de la culture même des gens. Si les citoyens d'un pays sont en mesure de comprendre, il faut le leur dire et arrêter de cultiver de manière subtile l'ignorance et le préjugé d'une incapacité à assumer les idéaux d'un Etat de droit, et partant la culture démocratique. ${ }^{30}$ Alors que bon nombre d'Etats reconnaissent, dans leurs constitutions, le droit des citoyens d'être informés, ${ }^{31}$ les gouvernants leur privent, par contre, toute communication législative pouvant les renseigner sur qu'ils doivent connaitre pour apporter leur à l'édification et à la consolidation d'un Etat de droit et donc un Etat démocratique.

Est-ce que les attributs définissant la démocratie peuvent-ils être conçus universellement sans tomber dans une sorte d'idéologie généralisable? La question, à mon avis, reste ouverte. Car dès qu'on part des prémisses erronées, l'on arrive, sans nul doute, aux conclusions fausses.

Pour ne prendre que l'exemple de la République Démocratique du Congo comme illustration de cette théorie, il ressort que l'on est démocrate que quand l'on est dans l'opposition. Une fois au pouvoir, l'on développe des théories de la conservation de celui-ci.

Car au-delà du fait que l'Etat de droit soit la pierre angulaire de la démocratie constitutionnelle contemporaine, les comportements pratiques des hommes politiques, Africains en

29 Les exemples font légion en Afrique de ces attitudes incongrues caractérisant les leaders politiques africains. Tout récemment, en Afrique australe, la présidente sortante du Malawi qui, après les élections jugées satisfaisantes par nombre d'observateurs et qu'elle perdait sûrement, a décrété l'annulation du scrutin. Dans bien des pays, l'on note des leaders qui déclarent, après les élections, l'illégitimité ou l'illégalité d'un vote auquel ils n'ont pas participé ou ayant participé, ils n'ont pas fait campagne. Tout cela constitue des bruits des syrènes destinés à d'autres cieux qu'aux Africains eux-mêmes.

30 Il existe un devoir public de consentir à tous les citoyens, et ce gratuitement, la possibilité de connaître les lois, les sentences et les actes gouvernementaux à travers les nouveaux instruments informatiques et télématiques aussi, et de les rendre aisément connaissables et trouvables de la part de toutes les composantes la collectivité. Un citoyen informé est essentiel pour la création d'un sain processus démocratique décisionnel. Et plus il sait des autorités qui le gouvernent et mieux il sera gouverné. Le devoir indéfectible du législateur est celui de faire en sorte que le commandement impératif contenu dans la loi ait le maximum de vulgarisation entre les citoyens afin d'amplifier la possibilité d'une son effective et consciente réalisation. La connaissance des lois et actes parlementaires est une ressource à promouvoir à tout prix, parce qu'elle représente une base essentielle pour la construction d'une conscience démocratique, civile, sérieuse et consciente.

31 Articles 22 et 23 de la Constitution de la République Démocratique du Congo du 28 février 2006. 
général et Congolais en particulier, prouve le contraire quant à la culture de la prise en compte des normes qui garantissent la pérennité des principes de la démocratie d'un pays donné. Et tout est fait selon les humeurs des nouveaux dirigeants. Et cela a comme conséquence la non continuité des actes administratifs, posés par les gouvernants précédents, alors qu'ils avaient encore la force leur attribuée en leur temps.

Dans beaucoup de pays, en Afrique notamment, sans pour autant faire fi de ce qui se passe ailleurs et même dans des pays de grande tradition démocratique-au sens occidental du terme-la culture de la conservation du pouvoir par un seul homme ou par un clan ne relève en rien d'une culture démocratique. Le fossé qui existe entre certaines dispositions constitutionnelles des pays africains, excluant formellement les liens parentaux ou amicaux comme critère d'attribution de postes de responsabilité étatique et les pratiques en cours ne s'inscrivent en rien dans une quelconque culture démocratique.

\section{Conclusion}

Parler de l'Etat de droit et de la démocratie constitue un défi qu'il fallait relever dans la mesure où ce sujet semble être sans objet dans l'entendement de la plupart des gens, alors qu'aujourd'hui, la réalité sur terrain prouve que l'expression d'Etat de droit et le concept de démocratie sont encore des notions à définition variable, selon que l'on est dans telle culture plutôt que dans telle autre.

L'existence des textes juridiques reconnaissant la pratique démocratique dans la gestion de la chose ne signifie absolument pas l'existence de la démocratie comme un état de fait. Car au-delà des textes, il faut une culture non seulement des textes, mais aussi du respect de ces mêmes textes. La démocratie est aussi la conscience d'une valeur, d'une valeur positive. Cette valeur est positive en ce sens qu'elle reconnaît positivement le bien fait par d'autres dans l'intérêt de la collectivité.

Pour moi les textes, dans l'instauration et la consolidation de la démocratie en vue de l'existence d'un Etat de droit, servent de support à la culture et à la conscience démocratique. Cette étude est importante parce qu'elle repense le rapport entre la reconnaissance d'un fait et son existence effective. Beaucoup de pays africains, en général, et la RDC, en particulier, se disent démocratiques et donc constitutifs d'un Etat de droit, alors qu'ils sont encore inscrits dans des processus de démocratisation, car la démocratie n'est pas un état de fait mais un état d'esprit, une valeur, une conscience et une culture. 